Musées, Patrimoine et Culture scientifiques et techniques

112 | 2007

juillet - août 2007

\title{
La musique et ses instruments au musée du quai Branly
}

\section{Madeleine Leclair}

\section{OpenEdition \\ Journals}

Édition électronique

URL : http://journals.openedition.org/ocim/739

DOI : 10.4000/ocim.739

ISSN : 2108-646X

Éditeur

OCIM

Édition imprimée

Date de publication : 1 juillet 2007

Pagination : 30-39

ISSN : 0994-1908

Référence électronique

Madeleine Leclair, "La musique et ses instruments au musée du quai Branly », La Lettre de l'OCIM [En ligne], 112 | 2007, mis en ligne le 15 février 2011, consulté le 19 avril 2019. URL : http:// journals.openedition.org/ocim/739; DOI : 10.4000/ocim.739 
d'Ethnographie du Trocadéro environ 150 instruments de musique dont la plupart étaient des pièces archéologiques. En 2000, elle comprenait environ 10000 instruments provenant des cinq continents. L'histoire de cette collection est déterminée par certains événements socio-politiques particulièrement importants en France, et par le développement de la recherche scientifique en Ethnologie et en Anthropologie. La chronologie du développement de cette collection peut se définir par la succession de quatre grandes périodes.

La première va de 1878 aux années 1910. L’idée de rassembler à Paris, en un même lieu, des objets ethnographiques disséminés en divers endroits se concrétisa en 1878, à l'occasion de l'Exposition Universelle qui eut lieu cette même année au musée d'Ethnographie du Trocadéro. Devenu l'une des vitrines importantes de la France, ce musée attira de nombreux voyageurs et collectionneurs qui, par des dons, contribuèrent à son rayonnement. D'importants dépôts furent également consentis par certaines institutions gouvernementales, muséographiques ou non, enrichissant ainsi les collections du musée à cette époque.

De 1910 à 1928, le musée d'ethnographie du Trocadéro a souffert d'un important manque de financement, ce qui s'est notamment traduit par la très faible croissance des collections instrumentales pendant ces années - environ 250 instruments de musique seulement sont entrés au musée au cours de ces 18 années. La recherche ethnographique n'était pas encore institutionnalisée, et le musée avait peu de moyens à investir dans le travail de collecte sur le terrain.

Pendant la troisième grande période, qui va de 1928 à 1940, la conjugaison de divers faits liés au développement de l'Ethnographie et à l'effervescence artistique de l'époque marque un tournant décisif dans l'histoire de la constitution des collections du musée d'Ethnographie du Trocadéro, qui deviendra le musée de l'Homme, ouvert au public en 1938. Nommé directeur du musée d'Ethnographie du Trocadéro en 1928, Paul Rivet, anthropologue américaniste, procéda au rattachement officiel de ce musée à la chaire d'Anthropologie du Muséum national d'Histoire naturelle. Devenu un lieu de rencontre pour la communauté scientifique, le musée se vit allouer des crédits financiers importants, grâce auxquels de très importantes missions de recherche et de collecte sur le terrain purent être envisagées. La sélection des objets s'opère désormais selon des critères scientifiques : les instruments de musique entrant au musée formeront désormais des séries culturellement significatives et cohérentes. Durant ces années, la collection des instruments de musique d'Afrique s'est enrichie de manière spectaculaire : le nombre d'instruments provenant de ce continent est en effet passé de 596 en 1926 à plus de 1800 en 1940. Cette époque, qui marque l'apogée de l'Ethnographie française, est celle de la célèbre mission «Dakar-Djibouti » (mai 1931-février 1933) conduite par Marcel Griaule et Michel Leiris, à laquelle a notamment participé André Schaeffner, musicologue et ethnologue, fondateur en 1939 du département d'Ethnologie musicale du musée de l'Homme ; elle est également celle d'un véritable engouement de la bourgeoisie parisienne pour le jazz et l'exotisme que représentaient les musiques africaines à l'époque.

Durant la période qui va de l'Après-guerre jusqu'en 2000, le laboratoire d'Ethnologie du musée de l'Homme et plus particulièrement son département d'Ethnomusicologie - au sein duquel une formation de recherche qui est à l'origine du laboratoire d'Ethnomusicologie aujourd'hui devenu le centre de Recherche en Ethnomusicologie, fut mise en place en 1968 par Gilbert Rouget - diversifie et multiplie ses activités de recherche et de diffusion, allant de pair avec un accroissement soutenu des collections, notamment celles qui proviennent des continents africain et asiatique. Parmi les événements les plus marquants de ces années, mentionnons la première grande mission ethnomusicologique, la mission «Ogooué-Congo » en Afrique équatoriale, qui fut organisée en 1946. André Didier, professeur au conservatoire national des Arts et Métiers, Gilbert

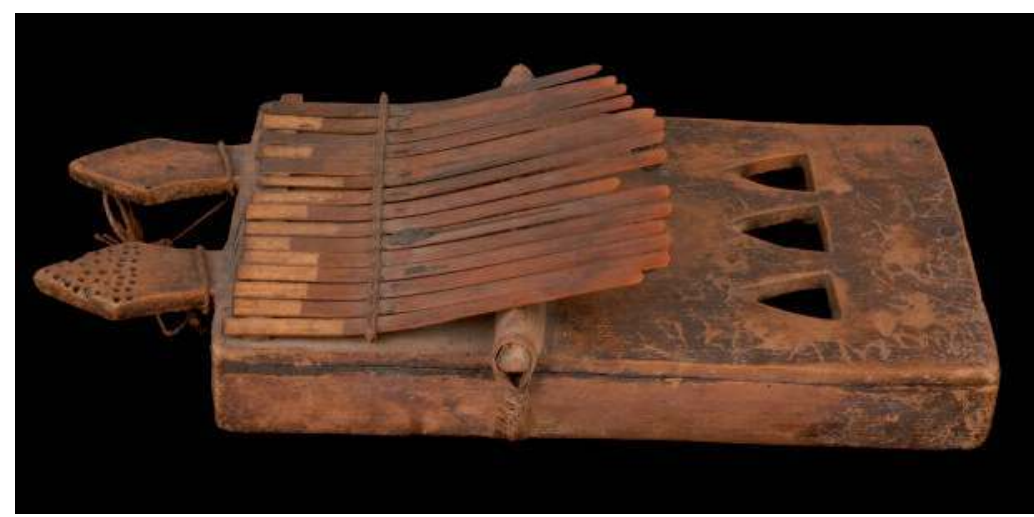

Lamellophone (timbiri) en bois de fromager et écorce de rônier, Sanaga du village de Nachtigal, Cameroun. Recueilli en 1931 (mission Dakar-Diibouti, ancienne collection du musée de l'Homme) ○ musée du quai Branly 


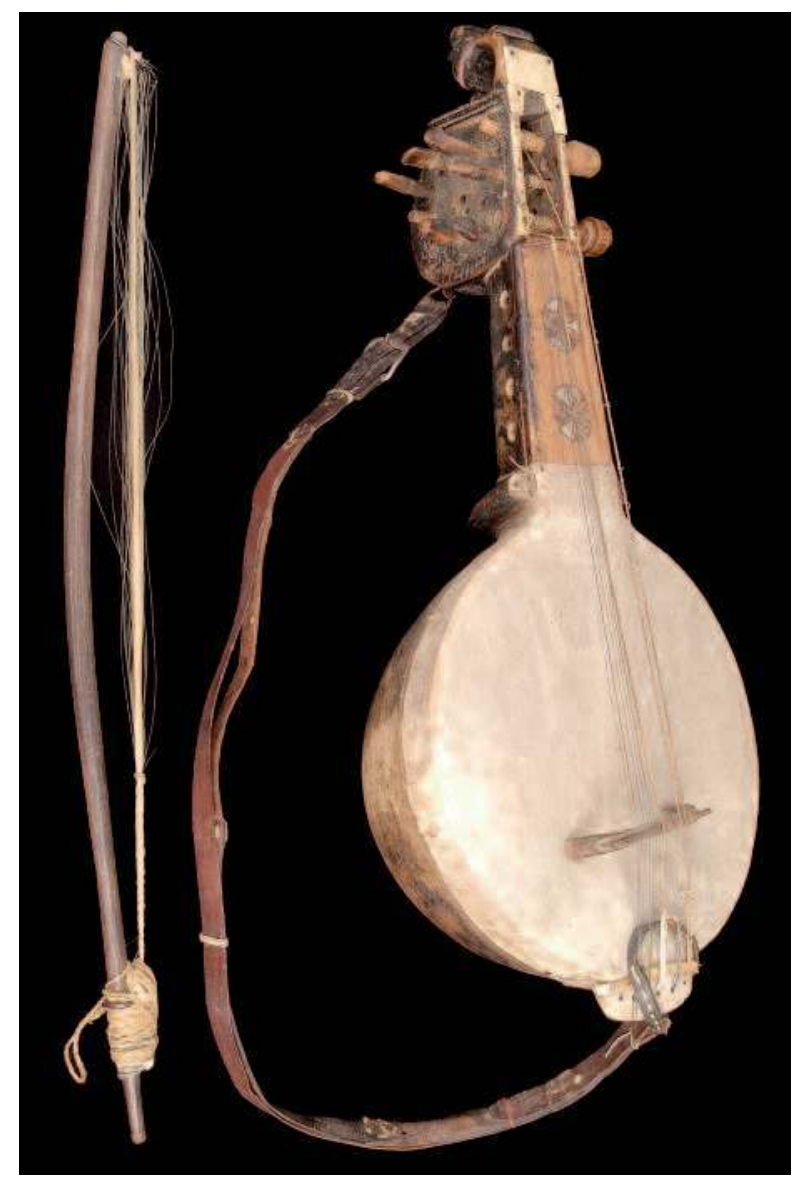

Vièle à 3 cordes (kamayacha) en bois, peau, crins, ivoire et nacre, désert de Thar, district de Jaisalmer, Rajasthan, Inde. Recueillie en 1976 (mission Geneviève Dournon, ancienne collection du musée de l'Homme) () musée du quai Branly

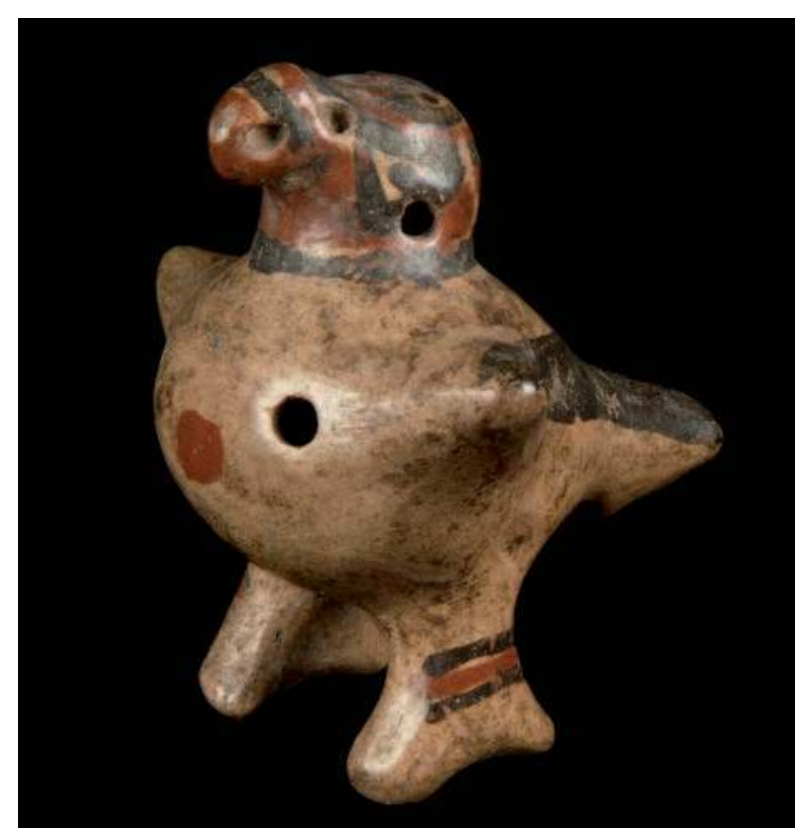

Flûte globulaire en terre cuite polychrome en forme d'oiseau, Panama, 1000 à 1500 (don Alphonse Pinart ancienne collection du musée de l'Homme) (c) musée du quai Branly
Rouget, ethnomusicologue, et l'explorateur PierreDominique Gaisseau, y enregistreront plus de 600 disques de gravure directe de musique bantou et pygmée. Quelques 200 instruments de musique du Cameroun et du Niger furent rapportés au musée par Marcel Griaule lors de ses deux importantes missions de recherche de 1938 et de 1959. L'enrichissement de la collection des instruments de musique d'Asie est, au cours de cette période, autant lié à d'importants dons et dépôts d'autres institutions muséographiques - musée Guimet, musée de la Marine et autres - qu'aux collectes faites lors de missions de recherche sur le terrain. Corneille Jest rapporta, entre 1961 et 1985, plus de 75 instruments du Népal et du Bhoutan. Geneviève Dournon, responsable de la collection d'instruments de musique du musée de l'Homme de 1967 à 2004, mena d'importantes missions de terrain dans les régions du centre et du nord de l'Inde entre 1972 et 1992, qui lui ont permis de recueillir plus d'une centaine d'instruments de musique.

Entre 2001 et 2004, les quelques 300 instruments de musique d'Europe furent regroupés puis transportés dans les réserves du musée national des Arts et Traditions populaires, en attendant de pouvoir rejoindre le futur musée des Civilisations de l'Europe et de la Méditerranée. Les instruments de musique d'Amérique et d'Asie, ainsi que les ensembles d'instruments provenant d'Afrique et d'Océanie, considérablement enrichis par les pièces provenant de l'ancien musée national des Arts d'Afrique, furent réunis. Inventoriée et entièrement numérisée, cette collection est maintenant conservée dans une réserve qui lui est spécialement dédiée.

\section{La réserve des instruments de musique}

L'un des gestes architecturaux particulièrement original et spectaculaire imaginé par Jean Nouvel pour le musée du quai Branly consiste en une tour de verre haute de 23 mètres, traversant toute la hauteur du bâtiment. Cette tour de verre, pièce maîtresse et centrale dans l'architecture du bâtiment, est perceptible dès le hall d'accueil du musée, et constitue l'une des toutes premières sollicitations du visiteur. Cette réalisation architecturale est d'autant plus forte qu'elle est dédiée à l'une des thématiques transversales du musée du quai Branly : la musique et ses instruments. En effet, cette tour est une réserve, un lieu où est conservée la collection des instruments de musique d'Afrique, d'Asie, d'Amérique, d'Océanie et d'Insulinde du musée. Traditionnellement immergée 
dans les profondeurs du bâtiment, cette réserve muséographiée et partiellement visible est intégrée au parcours muséographique et se déploie sur six niveaux pour une surface utile totale d'environ $620 \mathrm{~m}^{2}$. Près de 1900 mètres linéaires d'étagères et $190 \mathrm{~m}^{2}$ de parois grillagées destinées à des systèmes de rangement par suspension y sont installés. Les différents types de conditionnement de cette réserve ont été déterminés selon les normes et les exigences de la conservation préventive. L'hygrométrie, la température et le taux moyen annuel de luminosité sont contrôlés par des systèmes de climatisation et d'éclairage spécifiques.

L'objectif visé par la disposition architecturale de cette réserve et par le système de classement adopté est de présenter une vue d'ensemble des principaux types instrumentaux connus et joués sur chacun des quatre continents, des matériaux privilégiés pour leur construction, de la diversité des formes et des couleurs, mais aussi du travail que représentent, pour l'ethnomusicologue et le muséologue, la gestion et la conservation d'une telle collection.

Dans cette réserve au volume cylindrique, les instruments de musique sont regroupés selon l'aire continentale d'où ils proviennent, puis selon la famille organologique à laquelle chacun d'eux se rattache : aérophone, cordophone, membranophone et idiophone. Ces quatre grandes familles instrumentales sont ainsi dénommées en référence à la mise en vibration sonore d'un élément : mise en vibration de l'air pour les aérophones; vibration de cordes qui sont pincées, frottées ou frappées pour les cordophones ; une ou deux membranes frappées ou frottées pour les membranophones. Les idiophones forment une famille d'instruments pour lesquels la matière rigide dont est fait le corps de l'instrument est mise en vibration par entrechoc, frappement, secouement, pincement, raclement ou frottement.

Chacune de ces familles se subdivise en un certain nombre de types et de sous-types, selon la logique d'un système de classification dont l'élaboration s'est déroulée en plusieurs étapes, sur plus d'une centaine d'années, au fur et à mesure de l'accroissement considérable des collections instrumentales dans différents musées occidentaux, de la découverte d'instruments de musique inédits et du développement de la recherche en ethnomusicologie.

La source de ces travaux d'élaboration de systèmes de classification plonge dans un traité indien portant sur l'art dramatique et attribué au sage Bharata : le Natyasatra, rédigé en sanskit au début de l'ère chrétienne. Ce texte, véritable rhétorique de l'art, classifiait les instruments de musique indiens en quatre grandes catégories : tata (« étiré »), les instruments comprenant des cordes «étirées » ou tendues ; susira (« creux »), en référence aux instruments comportant un tube ou une colonne creuse, c'est-à-dire, pour Bharata, les instruments avec colonne d'air ; avanaddha (« couvert »), les instruments dont le corps est recouvert par une peau ; et ghana (« solide »), en référence à la densité de la matière dans laquelle l’instrument était fabriqué. C'est très probablement de cet ouvrage, donné au musée de l'Académie royale de Belgique en 1876 avec une centaine d'instruments de musique indiens par le Raja Sourindro Mohun Tagore, que s’inspirent les travaux d'organologie et de classification menés par Victor Mahillon, publiés une première fois en 1878 dans l'Annuaire du Conservatoire royal de musique de Bruxelles puis en 1880 dans le Catalogue descriptif et analytique du Musée instrumental du conservatoire Royal de Bruxelles. Erich M. von Hornbostel et Curt Sachs élargiront et préciseront ce système de classification en

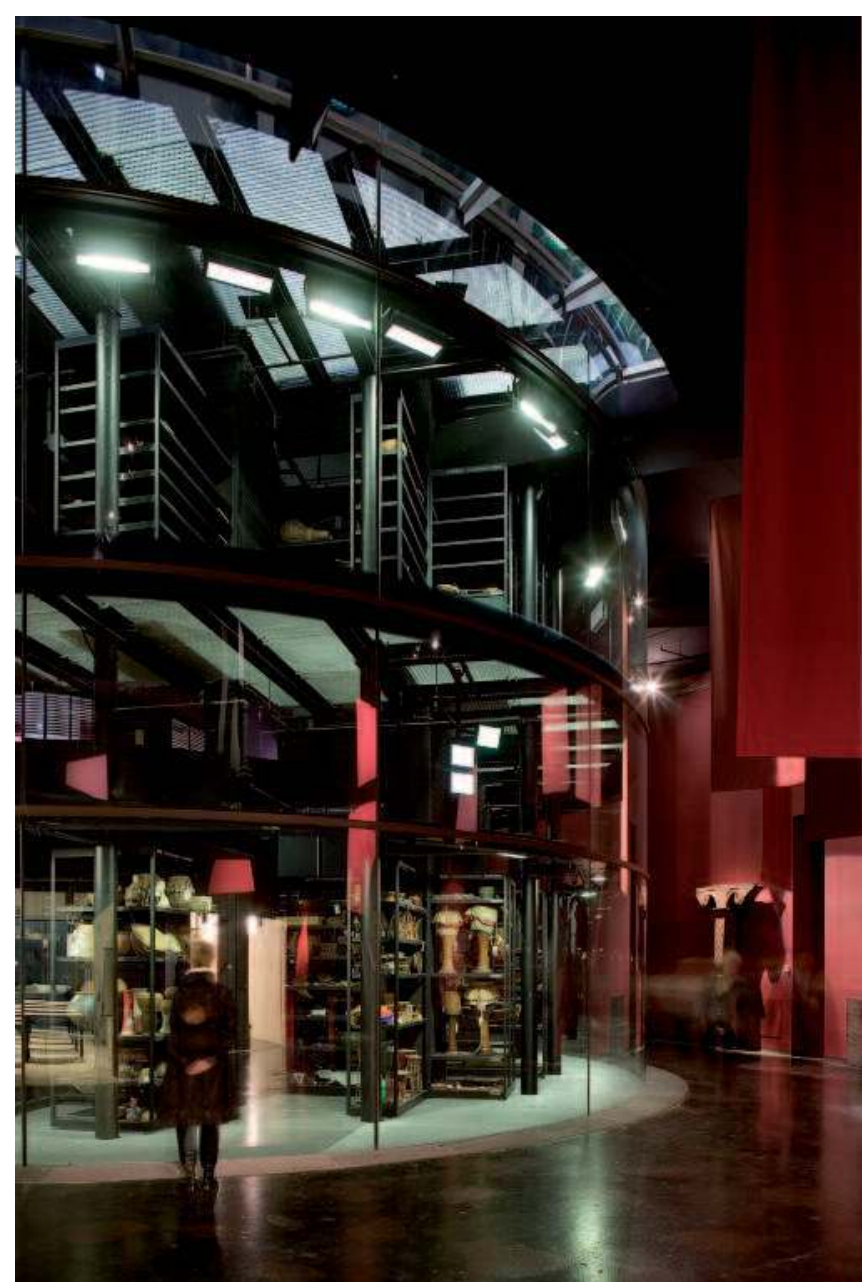

Vue des niveaux "Jardin Bas » et " Jardin Haut» de la réserve des instruments de musique, consacrés aux instruments d'Asie et d'Afrique. () musée du quai Branly/Nicolas Borel 
1914, dans un texte de référence - Systematik der Musikinstrumente : ein Versuch - qui, aujourd'hui encore, fait autorité. Au musée d'Ethnographie du Trocadéro puis au musée de l'Homme, André Schaeffner s'inspirera du travail de ses prédécesseurs pour imaginer un tout autre projet de classement, publié pour la première fois dans le Bulletin du musée d'Ethnographie du Trocadéro en janvier 1931 et qui propose de subdiviser l'instrumentarium en deux grandes familles : les instruments à corps solides vibrants, qui comprennent ceux qui sont constitués d'un corps solide non susceptible de tension (en pierre, coquillage, os, bois, terre cuite, métal, verre...) et ceux qui sont faits d'un corps solide susceptible d'être tendu (en écorce ou en peau), l'un et l'autre pouvant se présenter sous l'aspect d'une membrane ou d'une corde ; l'autre famille est formée par les instruments à air vibrant.

Le classement typologique qui fut appliqué à la collection instrumentale du département d'Ethnomusicologie du musée de l'Homme résulte de l'articulation des systèmes de classement proposés par C. Sachs,

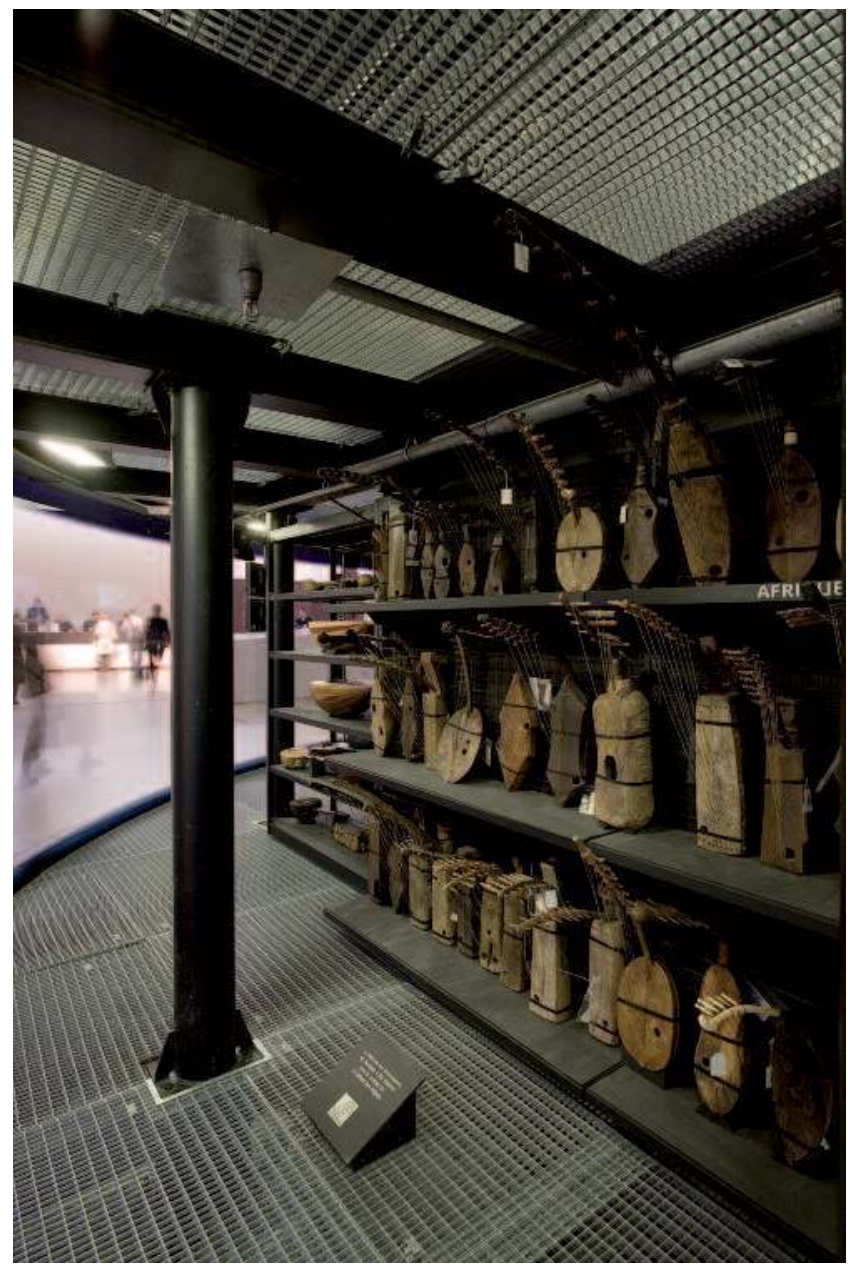

Niveau «Accueil » de la réserve des instruments de musique, où sont conservées les harpes arquées d'Afrique centrale. () musée du quai Branly/Nicolas Borel
E. M. von Hornbostel et A. Schaeffner. Il permet, en principe, de rattacher n'importe quel instrument de musique fonctionnant selon les lois de l'acoustique à l'une des quatre familles organologiques puis de distinguer, au sein de chacune d'elle, des sous-groupes instrumentaux identifiés selon que le procédé de mise en vibration de la matière est le frappement, le secouement, le raclement, le frottement, le pincement et le soufflement. Cette démarche de classement et la mise au point d'une terminologie spécifique se sont précisées au cours de travaux de recherche menés par Geneviève Dournon, chargée de la collection d'instruments de musique de cette institution de 1967 à 1995 , et par plusieurs autres chercheurs ethnomusicologues et organologues ayant travaillé sous sa direction.

C'est selon ce mode de classification que les 10000 instruments de musique conservés au musée du quai Branly ont été répartis sur les six niveaux de la réserve qui leur est destinée. La terminologique servant à les désigner et à les décrire a aussi été fidèlement respectée sur les différents supports de la signalétique, dans l'arborescence du thésaurus ainsi que sur les fiches de la base de données générale du musée.

Cette répartition des instruments selon le double critère de leur aire continentale de provenance et de leur type organologique a conduit à organiser la réserve instrumentale de la manière suivante :

- niveau «Jardin Bas »: environ 1800 instruments d'Asie appartenant aux familles organologiques des cordophones, des idiophones et des membranophones. Parmi les quelques 450 instruments à cordes, les principaux types sont les cithares, les luths et les vièles. Plus de la moitié des idiophones sont mis en vibration par frappement - gongs, tambours de bois, cloches, xylophones, lithophones et tambours de bronze. Les idiophones par entrechoc et par pincement - cymbales, cymbalettes et guimbardes - , largement diffusés en Asie, sont également bien représentés dans cette partie de la collection. Enfin, environ 300 membranophones asiatiques - des tambours sur caisse ou sur cadre, des timbales et quelques tambours à friction - sont également conservés à ce niveau de la réserve.

- niveau « Jardin Haut » : plus d'un millier d'instruments de musique d'Afrique et d'Asie. On compte environ 800 aérophones d'Asie, dont la moitié sont des instruments à anches, simples ou doubles. Parmi eux se trouvent les orgues à bouche (une centaine d'exemplaires), type instrumental spécifique à la zone Sud-Est du contient asiatique. Une partie de la vaste collection d'instruments à cordes d'Afrique se trouve également rangée à ce niveau. Elle comprend 
une centaine d'instruments à cordes frottées - les vièles - une centaine de cithares et une soixantaine de harpes à chevalet et de harpes-cithares, deux des formes selon lesquelles la vaste famille des harpes se décline sur le continent africain. Cette section de la réserve consacrée à l'Afrique conserve également une partie des idiophones par frappement : les tambours de bois et les xylophones.

- niveau «Accueil »: cordophones et aérophones d'Afrique. De toutes les familles organologiques, celle des cordophones est, en Afrique, la plus diversifiée. La diffusion de quelques types instrumentaux, comme les luths ou les arcs musicaux, concerne l'ensemble du continent. À l'inverse, certains cordophones ont prospéré dans des régions de l'Afrique plus ou moins vastes : les harpes arquées se trouvent principalement dans une zone qui part du Gabon à l'ouest et rejoint l'Ouganda à l'est, en passant au nord par la République centrafricaine et le Tchad, tandis que les harpes fourchues semblent n'être utilisées que dans le sud de la Côte-d'Ivoire et en Guinée. Ce niveau de la réserve accueille également un millier d'aérophones, en majorité des flûtes et sifflets ainsi que des trompes latérales et terminales.

- niveau « R1 »: membranophones et idiophones d'Afrique. Environ 1900 instruments de musique y sont conservés. Le tiers d'entre eux sont des tambours, qui occupent plus de la moitié de l'espace de rangement. L'aspect rudimentaire de certains idiophones d'Afrique peut parfois masquer une réelle inventivité dans l'usage qu'en font les musiciens : ainsi les tape-cuisses, simplement faits d'une longue courge de calebasse évidée et ouverte aux deux extrémités, se jouent en petits ensembles, par des musiciennes qui réalisent un véritable contrepoint rythmique.

- niveau « R2 » : instruments de musique d'Océanie et d'Insulinde. 1100 instruments de musique sont conservés sur ce niveau de la réserve, qui se trouve dans l'environnement muséographique du plateau de référence de la collection. Plus de la moitié des instruments de musique d'Océanie sont des aérophones : flûtes de Pan, flûtes terminales et latérales, flûtes nasales, conques, trompes et rhombes. La diversité de cette famille contraste avec celle des cordophones, au nombre d'une vingtaine seulement dans la collection et dont la facture est d'une grande sobriété. Les instruments de musique d'Insulinde se répartissent à peu près également entre les quatre grandes familles organologiques. Cette collection regroupe notamment une importante série de luths et de cithares dont le profil de la caisse rappelle celui d'un bateau.
- niveau « R3 » : instruments de musique d'Amérique. Les 2450 instruments de musique d'Amérique sont répartis en deux grands ensembles : ceux qui sont issus de fouilles archéologiques en MésoAmérique et ceux de facture plus récente. Parmi les 900 pièces préhispaniques se trouvent des aérophones : flûtes, flûtes de Pan, sifflets, conques et vases siffleurs, auxquels s'ajoutent des grelots et des hochets. Près du deux tiers des instruments de musique recueillis au cours de missions ethnologiques sur le continent américain sont des instruments à vent, la plupart étant des flûtes. Viennent ensuite les idiophones, de types très divers : cloches, hochets, carapaces de tortue frottées, crécelles, guimbardes, sonnailles, sistres. La famille de membranophones compte une centaine d'instruments, tandis que les instruments à corde sont les moins nombreux dans cette partie de la collection.

Si cette réserve partiellement visible est généralement bien accueillie, il n'en reste pas moins que sa lisibilité n'est pas immédiatement évidente pour l'ensemble du grand public, certains visiteurs découvrant l'existence de réserves dans un musée, ou sont en tous cas confrontés pour la première fois à ce type de lieu muséographique, pourtant essentiel.

\section{L'exposition de la musique et de ses instruments : de la mise en scène $d$ 'une vitrine à la mise en son d'un espace}

La musique et ses instruments au sein des parcours géographiques du musée

Plus d'une centaine d'instruments sont exposés sur le plateau de référence du musée. Dans les espaces dédiés aux arts et aux cultures d'Amérique, d'Asie, d'Insulinde, d'Océanie, et dans certaines vitrines consacrées à l'Afrique, ces instruments prennent place parmi d'autres objets, et contribuent à la mise en scène d'un propos muséographique extra-musical. Cinq groupes instrumentaux ponctuent également le parcours muséographique de l'Afrique, en alternance avec une autre thématique transversale : les textiles. Regroupant au total une cinquantaine de pièces, ces ensembles comprennent certains instruments de musique de la vaste région du Maghreb et du Sahel, et quelques instruments représentatifs des quatre grandes familles organologiques.

Le musée dispose, en plus des objets disposés en ou hors vitrine, d'un véritable système d'information multimédia, diffusant près d'une centaine de programmes. Parmi eux, environ 80 programmes dits de 


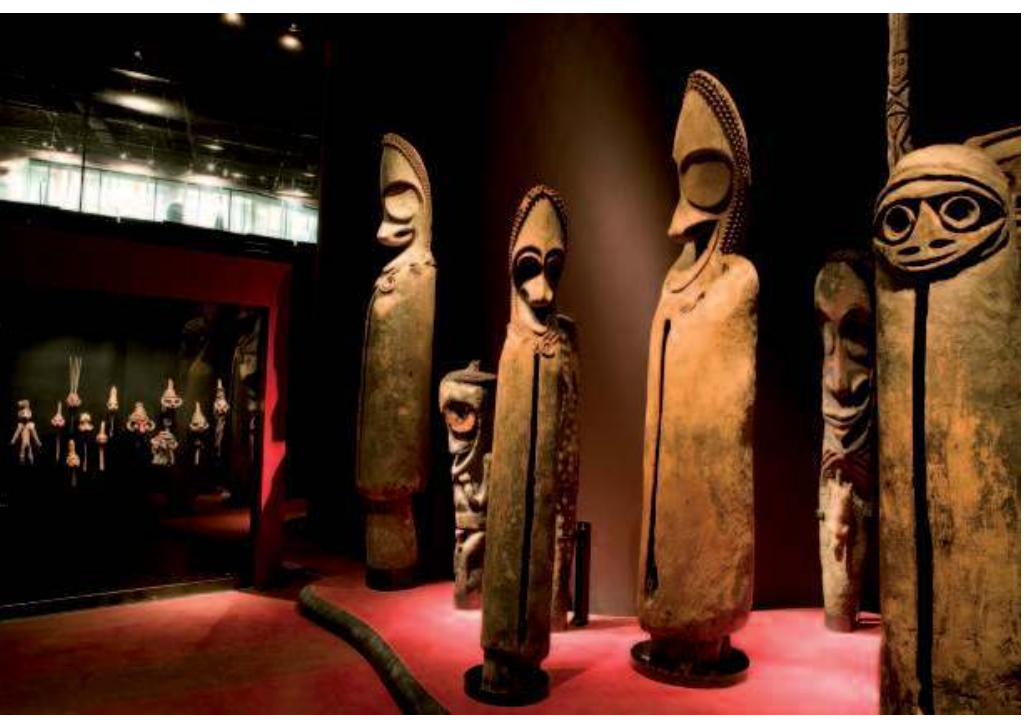

Tambours de bois des îles d'Ambrym et de Malekula au Vanuatu (Mélanésie), exposés dans la section «Océanie » du plateau de référence du musée du quai Branly. Taillés dans des troncs d'arbre évidés et destinés à être frappés au moyen de bâtons de bois ou de pierres, ces instruments de musique étaient disposés en bordure de vastes places de danse. ( ) musée du quai Branly/Antoine Borgeau

niveau 1, "sont directement liés aux objets présentés dans la vitrine sur laquelle ils sont disposés. Leur rôle premier consiste à présenter des ceuvres ou des documents ethnologiques permettant de contextualiser les objets attenants dans leur environnement géoculturel » (Bezombes, 2006, p. 10).

Ainsi, les sections consacrées à Madagascar et à l'Ethiopie sont sonorisées : des musiques habillent l'espace et y jouent un rôle de repère sensoriel, de mise en condition de l'esprit pour découvrir de petits lieux muséographiques dédiés. Pour quatre vitrines du secteur Afrique, une bande sonore composée d'une suite d'extraits de pièces de répertoire est diffusée à proximité des instruments exposés. Ces dispositifs visent à faire découvrir simultanément le double potentiel artistique d'un instrument : sa plastique et sa contribution à un événement musical.

Enfin, l'un des programmes multimédia de niveau 1 présente un film sonorisé consacré à une importante danse rituelle des Peuls du Niger, tandis qu'un autre est consacré à l'art des Pygmées d'Afrique centrale, et notamment à leur musique vocale et à certaines de leurs danses rituelles. Dans ces deux exemples, c'est donc par le biais de la musique et de la danse que les visiteurs sont invités à découvrir le patrimoine artistique de ces populations.
Des espaces spécifiquement dédiés à l'exposition de la danse et de la musique

Le développement de la muséographie portant sur la culture immatérielle s'est fait en parallèle avec le développement des technologies du son et de l'image. L'élaboration d'un projet muséographique prenant comme objet la musique et la danse résulte d'un travail de collaboration entre une vingtaine de chercheurs ethnomusicologues et les membres d'un groupement pluridisciplinaire No design, spécialisé dans la production d'œuvres multimédia. Il prend la forme de trois installations multimédia disposées autour de la réserve des instruments de musique, sur le plateau de référence et sur la galerie centrale d'information anthropologique du musée. Ces installations sont autonomes mais complémentaires, tant du point de vue de l'articulation des contenus que des dispositifs mis en place.

Les principes muséographiques sous-jacents à ce projet sont de rendre sensibles des données abstraites, d'éviter l'exhaustivité pour privilégier la représentativité, de proposer un rapport personnel à la musique et aux musiciens, de susciter l'émotion plutôt que l'étonnement, en créant des lieux spécifiques permettant une rencontre entre un patrimoine musical et un nombre raisonnable de visiteurs et de musiciens.

\section{Autour de la réserve des instruments de musique}

Une série de dispositifs audiovisuels est répartie autour de la réserve. Ces dispositifs consistent en une dizaine d'ensembles d'écrans installés à l'intérieur de la tour de verre. Ceux-ci servent de support à la projection d'images fragmentées, évoquant de manière stylisée le jeu de certains instruments. Ces images sont associées à un système de diffusion d'extraits de pièces musicales représentatives. La diffusion sonore se fait par fixation de transducteurs contre les parois de la tour, utilisant la surface du verre comme s'il s'agissait de la membrane vibrante d'un haut-parleur.

L'enjeux de cette installation multimédia à la fois innovante et discrète est de construire un lien entre l'esthétique formelle des instruments de musique, déterminée aussi bien par le contexte d'exécution des répertoires musicaux que par la diversité des matériaux disponibles pour leur construction, et l'esthétique musicale recherchée par les facteurs instrumentaux qui ont su développer avec ingéniosité une multitude de dispositifs et de mécanismes de jeu. 


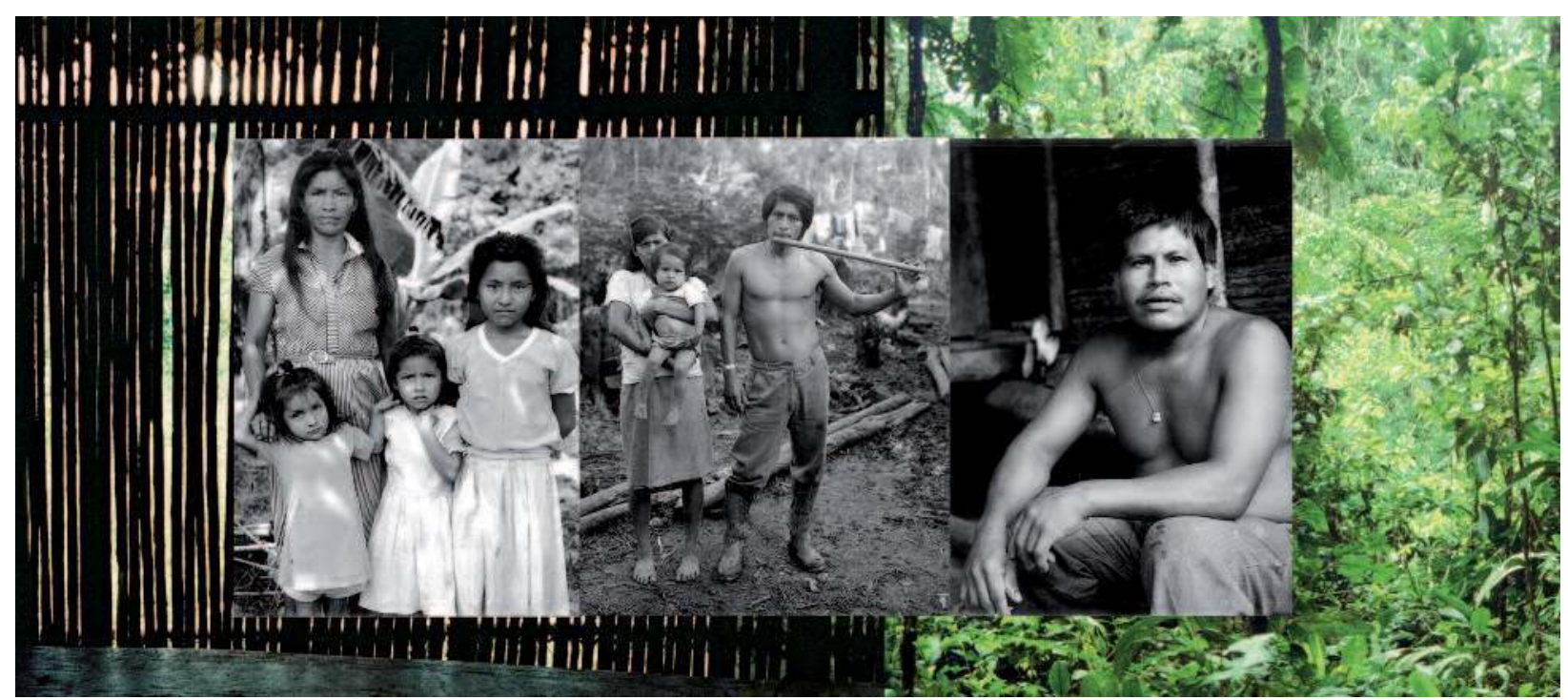

Imelda Sharup et sa famille, Kukush et sa famille, Rafael Tseremp : population Shuar (Jivaro) installée à Taruka en Équateur (1994). Le programme Musique et espaces en Amazonie du nord-ouest, diffusé dans la Boîte à musique Ouest, est consacré à l'univers sonore de cette population. (c) Pierre-Jérôme Jehel

\section{Les Boîtes à musique Est et Ouest}

Les Boîtes à musique Est et Ouest mettent en avant le caractère immanent de la musique, associant la danse, les composantes acoustiques de la musique et ce qui relève de ses conditions d'existence. Les Boîtes à musique sont deux espaces d'environ $35 \mathrm{~m}^{2}$, situés sur le plateau de référence du musée. Au total, 9 programmes d'environ six à dix minutes chacun y sont diffusés successivement, selon un ordre pré-établi (quatre dans la Boîte à musique Ouest et cinq dans la Boîte à musique Est). Lintention initiale était de laisser ces espaces totalement vide d'objets, laissant aux visiteurs la possibilité de circuler et de se déplacer librement. Or, l'expérience montre qu'en l'absence de sièges, les visiteurs hésitent à occuper l'espace. Quelques pièces de mobilier destinés à ces Boîtes sont donc en cours de réalisation.

Les Boîtes à musique sont des lieux vivants de partage, de contact direct avec la musique et la danse. Elles proposent une expérience collective de la musique, mise en volume par une installation multimédia associant des dispositifs de spatialisation du son et la projection d'images immersives de grand format, aptes à déployer une thématique dans un espace tridimensionnel, ce qu'aucun autre support de communication n'est à même de transmettre avec autant de cohésion et de pertinence. L'enjeu principal des 9 programmes qui y sont montrés est de proposer une muséographie du sensible, de tenter la juxtaposition d'un parcours de l'œil et de l'oreille : l'image donne au son des sens nouveaux et vice versa.
Ainsi, les programmes ne sont pas construits selon le plan d'un scénario linéaire de type narratif.

La première Boîte à musique, située du côté Est du plateau de référence, accueille quatre programmes qui se proposent de mettre en valeur la manière dont certaines musiques servent à déterminer ou à qualifier un espace géographique, ou encore des programmes pour lesquels l'environnement géographique est une donnée pertinente pour l'analyse de la perception d'une musique. L'un de ces programmes porte sur des cérémonies annuelles ngaanyka des Peuls nomades wodaabe du Niger, au cours desquelles l'initiation des jeunes hommes met en scène une véritable guerre rituelle dont les seules armes sont le chant polyphonique et la danse. L'enjeu de cette confrontation musicale consiste à séduire les femmes du lignage adverse. Les mariages noués à l'issue de la cérémonie renforceront les relations entre lignages autour de nouveaux liens de parenté. Le second programme présente une suite de moments musicaux tirés de la grande cérémonie d'échanges nekowiar, pratiquée par les habitants du Middle Bush de lî̂le de Tanna au Vanuatu. Un troisième programme intitulé Musique et espaces en Amazonie du nord-ouest concerne un monde où s'entrecroisent différents univers sonores : dialogue de visite dans une maison, arc musical, rituels collectifs, «bruits » quotidiens du village, sons de la forêt. Le quatrième programme est consacré à des danses masquées (dyoh pyakkhan) et musiques de processions des cités royales népalaises. 


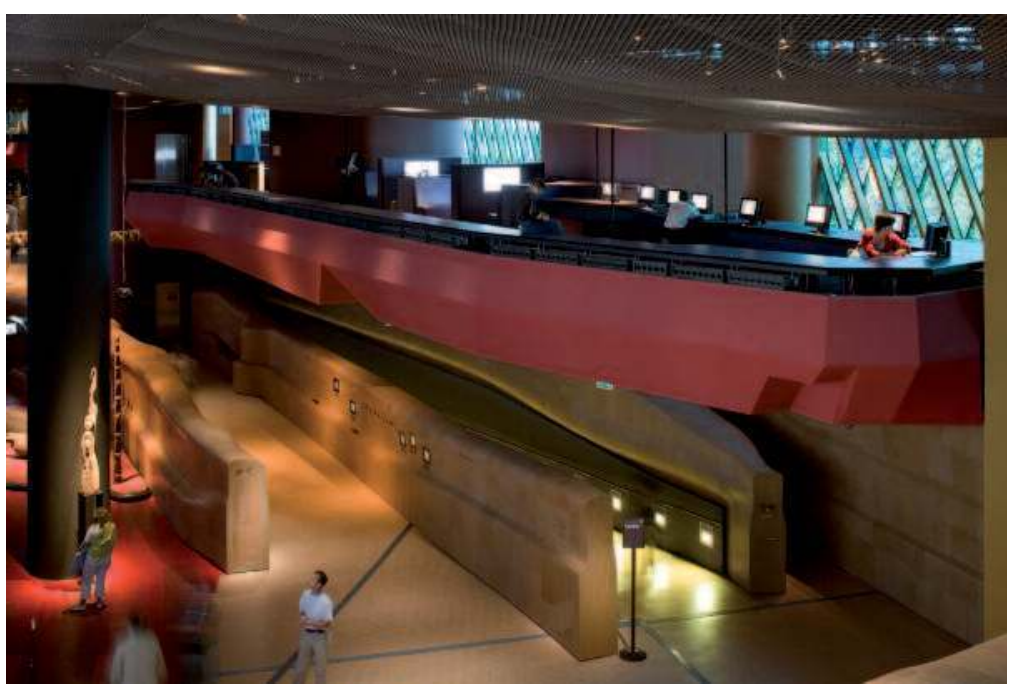

Le programme À l'écoute des musiques du monde, en cours de réalisation, pourra être consulté sur le Galerie multimédia. Suspendue au dessus du plateau de référence du musée, cette galerie est aussi un lieu de diffusion de l'ensemble des productions multimédia de niveau 1. (C) musée du quai Branly

Dans la seconde Boîte à musique sont projetés cinq programmes qui explorent le lien entre ceux qui participent à une même activité musicale, musiciens, danseurs ou spectateurs. L'un d'eux porte sur les polyphonies vocales des Pygmées Bedzan du Cameroun. Le second réactualise la scène d'un concert où se sont produits des musiciens iraniens. Un troisième programme permet de voir et d'entendre des chanteurs de basse caste Pulluva (Kérala, Inde du Sud), effectuant un rituel propitiatoire pour les divinités serpents et qui, par une double expression visuelle et sonore, invitent les divinités à se manifester. Le quatrième programme a comme sujet la musique et la danse des porteurs de masque, pendant la nuit qui clôture le rituel de gèlèdè chez les Itcha du Bénin, et le cinquième la grande fête bua' des Toraja Sa'dan de l'île de Sulawesi (Indonésie), un rituel destiné à la fécondité globale au cours duquel plusieurs chœurs chantent simultanément pour appeler l'énergie vitale perdue avec la mort.

Chacun des programmes est ainsi dédié à un événement musical particulier présenté dans son contexte d'exécution, et traité de manière à tenter d'évoquer la représentation que se font les musiciens de leurs propres pratiques.

Ces espaces totalement vides d'objets en trois dimensions se démarquent de tous les autres lieux muséographiques du musée, et semblent être particulièrement remarqués du public. Mais seule une enquête menée auprès des visiteurs nous dira si la

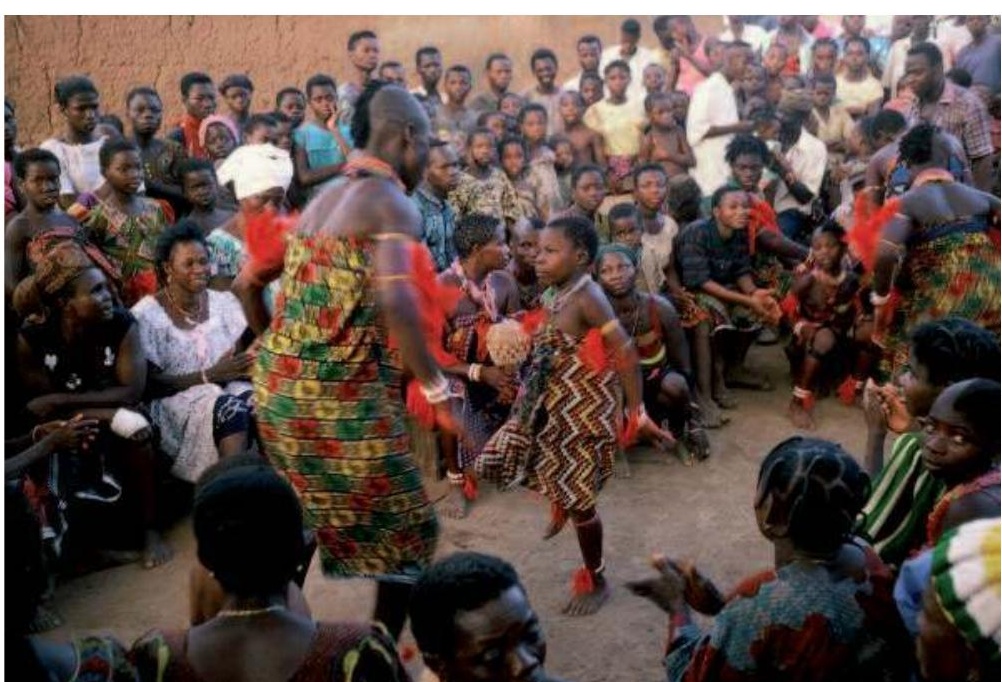

Danse des initiés au culte de Shampanon, divinité ayant le pouvoir de contrer les maladies contagieuses. Village de Koko, République du Bénin, population Itcha (1996). L'un des chapitres du programme $A$ l'écoute des musiques du monde présenté dans l'un des ateliers de la Galerie multimédia et consacré aux musiques initiatiques chez les Itcha.

(c) Madeleine Leclair

ligne éditoriale qui a été choisie pour la production des programmes est réellement perçue, et si l'intention des équipes de réalisation est comprise.

\section{La Galerie multimédia}

Enfin, deux autres lieux dévolus au temps de l'écoute sont actuellement en cours de préparation. L'un d'eux offrira au public une sélection de pièces musicales emblématiques de certains grands répertoires musicaux pratiqués sur les quatre continents. Une série de dispositifs multimédia sera également aménagée sur la galerie centrale d’information anthropologique du musée. Ils offriront un aperçu de la diversité des esthétiques musicales dans le monde et suggéreront des pistes d'écoute et de compréhension qui mettent l'accent sur le savoir musical, par un mode de visualisation de la musique mettant en lumière la forme et la nature de certains matériaux musicaux.

Ce projet expérimental d'exposition de la musique et de la danse dans les espaces muséographiques du musée du quai Branly se matérialise donc par la réalisation des différents types d'installations multimédia qui viennent d'être rapidement décrits. Il offre aux visiteurs plusieurs types d'expériences à valeur heuristique complémentaire : observer visuellement et auditivement quelques instruments de musique, vivre une expérience musicale unique dans les deux Bô̂tes à musique, et comprendre certains aspects fondamentaux des pratiques musicales, considérées dans leur contexte de production. 


\section{Bibliographie}

Bezombes, S. L'ethnologue, le musée et le multimédia. Produire et exposer le patrimoine immatériel au musée du quai Branly. Site Internet Réciproque. Produire et exposer l'immatériel, www.reciproque.net/articles/. 2006, 18 p.

Dias, N. Le musée d'ethnographie du Trocadéro : 1878-1908. Anthropologie et muséologie en France. Paris : Éditions du CNRS, 1991, 310 p.

Dournon, G. L'héritage muséographique d'André Schaeffner : les collections d'instruments de musique du musée de l'Homme, in Les fantaisies $d u$ voyageur. XXXIII variations Schaeffner. Paris : Société française de Musicologie, 1982, pp. 214-220.

Dournon, G. Le Salon de musique du Musée de l'Homme. Instruments traditionnels et programmes musicaux, édition revue et augmentée. Paris, Muséum National d'Histoire Naturelle, 1993, 16 p.

Dournon, G. Guide pour la collecte des musiques et instruments traditionnels. Paris : UNESCO, 1996, $151 \mathrm{p}$.

Grognet, F. D'un Trocadéro à l'autre, histoire de métamorphoses. Mémoire de DEA, Muséum national d'Histoire naturelle, 1997-1998.

Hornbostel, E. et Sachs, C. Systematik der Musikinstrumente : ein Versuch, Zeitschrift für Ethnologie, XLVI, 1914, pp. 553-590.

Leclair, M. Musique, in Guide du musée du quai Branly. Paris : Éditions du musée du quai Branly, 2006, pp. 272-277.
Leclair, M. Musique au musée, TDC (Textes et documents pour la classe), $n^{\circ} 918,2006$, pp. 18-21

Leclair, M. Les collections d'instruments de musique au musée du quai Branly, Cahiers de musiques traditionnelles, $n^{\circ} 16,2003$, pp. 30-35

Mahillon, V.-C. Catalogue descriptif et analytique du Musée instrumental du conservatoire Royal de Bruxelles. Précédé d'un essai de classification méthodique de tous les instruments anciens et modernes. Gand : C. Annoot-Braekmann, 1880, 395 p.

Rouget, G. Département d'ethnomusicologie du Musée de l'Homme, Revue de Musicologie, n54 (1), 1973, pp. 25-33.

Schaeffner, A. Origine des instruments de musique. Introduction ethnologique à l'histoire de la musique instrumentale. Paris : Éditions de l'École des Hautes Études en Sciences Sociales, 1994, 511 p.

Schaeffner, A. Projet d'une classification nouvelle des instruments de musique, in Le sistre et le hochet. Musique, théâtre et danse dans les sociétés africaines. Hermann, collection Savoir / Cultures, 1990, pp. 147-154.

Schaeffner, A. Le jazz (avec la collaboration d'André Cœuroy, préface de Franck Ténot). Paris : Éditions Jean-Michel Place, collection Les cahiers de Gradhiva n², 1988, 170 p. 